\title{
Digital Simulation Assessments of the Sensitivity of Quantitative MRI for Detection of an Iron Oxide Nanoparticle Brain MRI Contrast Agent
}

Joong Kim

Henry M. Jackson Foundation

Wen-Tung Wang

Henry M. Jackson Foundation

Andrew Knutsen

Henry M. Jackson Foundation

David Brody ( $\square$ david.brody@usuhs.edu )

Uniformed Services University of the Health Sciences

\section{Research Article}

Keywords: R1 map, R2 map, MP2RAGE, IONP, Image co-registration, Relaxation

Posted Date: August 13th, 2021

DOI: https://doi.org/10.21203/rs.3.rs-798379/v1

License: (c) (i) This work is licensed under a Creative Commons Attribution 4.0 International License. Read Full License 


\section{Abstract}

There have been substantial efforts to develop targeted exogenous MRI contrast agents to assess specific brain pathologies. In parallel with other efforts, it is important to assess the sensitivity of candidate MRI methods for detection of contrast agents. Here, we propose a digital simulation approach, which includes MR relaxation (R1 and R2) mapping and image co-registration. We simulated the effects of $3 \mathrm{~nm}$ iron oxide nanoparticles (IONPs) as a model contrast agent. Two independent relaxation maps acquired from the brain of the same subject were co-registered. The baseline subtraction between the two relaxation maps showed good agreement, demonstrating the high reproducibility of the method. Next, the second relaxation map was digitally altered ("seeded") to simulate additional MR relaxation values corresponding to several concentrations of $3 \mathrm{~nm}$ IONPs in various locations. The maps of absolute differences between the first relaxation map and the digitally altered second relaxation maps were assessed for conspicuity. Results based on living mouse and human brains scanned at 9.4 T and 3.0 T respectively both indicated reliable conspicuity for signal equivalent to $0.06 \mathrm{mM}$ IONP or higher. Overall, the digital simulation approach is a useful method to improve the development of MRI contrast agents and accompanying MRI methodologies.

\section{Introduction}

Magnetic resonance imaging (MRI) has been widely used as a diagnostic tool in both preclinical and clinical studies. Its high spatial resolution allows MRI to examine the central nervous system (CNS) with great anatomical detail ${ }^{1-5}$. The intrinsic biological characteristics of brain tissue can be characterized using the MR properties of longitudinal relaxation (T1) and transverse relaxation (T2) times; these are commonly referred to as rates R1 (1/T1) and R2 (1/T2). Typically, R1 and R2 MRI provide differentiation of brain tissues ${ }^{1,5-9}$ and detection of abnormalities ${ }^{10-14}$. However, both R1 and R2 MRI still suffer from the lack of pathophysiological specificity. Thus, multiple research groups are approaching the problem of pathophysiological specificity by developing targeted exogenous contrast agents, such as iron oxide nanoparticles (IONPs) ${ }^{15-17}$.

It is well known that IONPs have both $\mathrm{R} 1$ and $\mathrm{R} 2$ shortening effects ${ }^{18}$, providing positive and negative MR signal enhancement, respectively. IONPs not only produce sensitive MR contrast but also provide a scaffold for conjugation of antibodies or other molecular targeting domains which may be used to provide pathophysiological specificity in brain ${ }^{19,20}$. In general, quantitative R1 and R2 mapping have advantages over T1 and T2 weighted images by providing quantifiable MR parameters with reduced variability from MR hardware differences, positioning in the scanner, and other factors. Given that T1 imaging is quite sensitive to the effects of applied contrast agents, there have been efforts to develop MR methodologies to quantitatively detect the R1 relaxation enhancing effects induced by IONPs ${ }^{21-24}$. Among these, the Magnetization Prepared - RApid Gradient Echo (MP-RAGE) with 2 inversion times (MP2RAGE) was chosen for R1 mapping in this study for several reasons: MP2RAGE is readily available 
on both human MRI scanners and preclinical scanners, the method is robust to RF field inhomogeneity, and it has no requirement for extensive post acquisition image processing ${ }^{21}$.

Ultimately, the effect of IONPs on longitudinal and transverse MR relaxation should be tested in living tissues such as the brain. To do this, the employed contrast agent must be delivered to brain tissue by passing through the blood-brain barrier (BBB). There have been many efforts to develop methodologies to safely carry MR contrast agents across the $B B B{ }^{25-29}$, but at present this remains a challenge. In the meantime, as BBB crossing methods are being developed, it is also important to assess the sensitivity of candidate MRI methods for detection of IONPs. In this way, we will gain a better understanding of the amount of IONPs that will have to be delivered across the BBB to yield a detectible signal in vivo.

Here, we propose a digital simulation method, which includes both R1 and R2 mapping with image coregistration, to evaluate the MR sensitivity of extremely small IONPs for both human and mouse brain. In order to mimic the hypothetical effects of several types of human brain pathology, the digital simulations were focused on brain regions which are well known to be vulnerable to neurological disease or injury. The cortex of rodent brain shows various pathologies in both transgenic and brain trauma animal models ${ }^{30-33}$. The hippocampus is well known to be vulnerable in multiple neurological diseases including Alzheimer disease, mesial temporal sclerosis, herpes simplex virus encephalitis, and many more 34-37. The depths of cortical sulci are the pathognomonic anatomical locations for the pathology that defines Chronic Traumatic Encephalopathy $(\mathrm{CTE})^{38}$, likely due to damage from shear deformation induced by rapid rotational acceleration and impact ${ }^{39} 40$. Thus, we focused on cortex in mice, and on hippocampus and cortical sulcal depths in humans.

\section{Results}

\section{Digital simulation workflow}

The main aim of the study was to establish a digital simulation method to test the sensitivity of proposed $\mathrm{R} 1$ enhancing MR contrast agents on the in vivo brain MRI. The digital simulation method is outlined in Fig. 1. The digital simulation required two independent R1 maps (Fig. 1a and b) of the same subject within a short time interval to reduce the likelihood that there will be any physiology-induced changes. The second scan R1 map (Fig. 1b) was co-registered to the first scan R1 map (Fig. 1a) using ANTs (http://stnava.github.io/ANTs/). Then, the absolute $\Delta \mathrm{R} 1$ (| $\Delta \mathrm{R} 1 \mid)$ map between the 1st R1 map (Fig. 1a) and the co-registered 2nd R1 map (Fig. 1c) was calculated to produce a baseline $|\Delta R 1|$ map (Fig. 1d). The baseline $|\triangle R 1|$ map had mostly zero values, $0.009 \pm 0.002(n=5$ mean $\pm S D)$, except brain tissue and ventricle border. This demonstrated that the two independent R1 maps were obtained without imaging artifact and that the co-registration was effective at a voxel-by-voxel level. The co-registered 2nd R1 map was then digitally altered ("seeded") to simulate additional R1 signals equivalent to those expected from an R1 enhancing contrast agent (Fig. 1e). The digitally added R1 values in Fig. 1e were 0.0265 (i), 0.053 (ii), 0.0795 (iii), 0.106 (iv), and 0.1325 (v) $\mathrm{s}^{-1}$, which are equivalent to $5,10,15,20$ and $25 \%$ of the in vivo 
mouse brain cortex R1 value of $0.53 \mathrm{~s}^{-1}$. Finally, the absolute $\Delta \mathrm{R} 1(|\Delta \mathrm{R} 1|)$ map between the 1st R1 map (Fig. 1a) and the digitally seeded 2nd R1 map co-registered to the 1st R1 map (Fig. 1e) was calculated to produce a digitally seeded $|\Delta R 1|$ map (Fig. 1f). The digitally added R1 enhancements were clearly visible in the $|\Delta R 1|$ map, though the conspicuity was modest for the less enhanced regions (i and ii).

\section{$\mathrm{R} 1$ of in vivo mouse brain and $3 \mathrm{~nm}$ IONP at both $4.7 \mathrm{~T}$ and 9.4 T.}

In vivo mouse brain $\mathrm{R} 1$ maps were obtained at both $4.7 \mathrm{~T}$ and $9.4 \mathrm{~T}$, with five mice each per scanner (Supplementary Fig. 1a - j) The five mice scanned at 9.4 T were the same subjects from our previous report ${ }^{41}$. Region of interest (ROI) analysis was performed on dorsal cortex, and the quantified $\mathrm{R} 1$ values were $0.62 \pm 0.01 \mathrm{~s}^{-1}\left(4.7 \mathrm{~T}, \mathrm{n}=5\right.$, mean \pm standard deviation) and $0.53 \pm 0.01 \mathrm{~s}^{-1}(9.4 \mathrm{~T}, \mathrm{n}=5$, mean \pm standard deviation). The relaxivity ( $\mathrm{r} 1$ ) of $3 \mathrm{~nm}$ IONP were assessed at both magnetic fields resulting in $1.87 \mathrm{mM}^{-1} \mathrm{~s}^{-1}$ at $4.7 \mathrm{~T}$ and $0.79 \mathrm{mM}^{-1} \mathrm{~s}^{-1}$ at $9.4 \mathrm{~T}$ (Supplementary Fig. 1k.). Thus, from an intrinsic relaxivity perspective, $4.7 \mathrm{~T}$ imaging has substantial advantages over $9.4 \mathrm{~T}$ imaging. These longitudinal relaxivities were used to simulate additional $\mathrm{R} 1$ signals for both $4.7 \mathrm{~T}$ and $9.4 \mathrm{~T}$.

\section{Reproducibility of in vivo mouse brain $\mathrm{R} 1$ map at both $4.7 \mathrm{~T}$ and $9.4 \mathrm{~T}$.}

As described in the digital simulation workflow, two independent in vivo mouse brain R1 maps were obtained from 5 mice within a one-week time interval. The 2nd R1 maps were co-registered to the 1st R1 maps and the absolute R1 difference maps (| $\Delta \mathrm{R} 1 \mid)$ were calculated (Supplementary Fig. 2a-j.) These baseline $|\triangle \mathrm{R} 1|$ maps provided quantitative assessments of the reproducibility of the combined MP2RAGE image formation and co-registration process with ANTs. The reproducibility was clearly higher at $9.4 \mathrm{~T}$ than at 4.7 T. The signal to noise ratio (SNR) of the 2nd inversion recovery T1 weighted image of MP2RAGE was substantially higher at 9.4 $\mathrm{T}$ compared with $4.7 \mathrm{~T}$ as well (Supplementary Fig. 2aa-jj). In the cortex, the $|\Delta \mathrm{R} 1|$ at $4.7 \mathrm{~T}$ was highly variable $(0.03 \pm 0.02)$ compared to $9.4 \mathrm{~T}(0.01 \pm 0.01)$ (Supplementary Fig. $2 \mathrm{k}$ vs. I). Thus, from an imaging reproducibility perspective, $9.4 \mathrm{~T}$ imaging has substantial advantages over $4.7 \mathrm{~T}$ imaging.

\section{Assessment of sensitivity of MP2RAGE derived R1 maps to simulated $3 \mathrm{~nm}$ IONP at 4.7 T vs 9.4 T.}

The sensitivity of MP2RAGE derived in vivo mouse brain R1 maps to simulated $3 \mathrm{~nm}$ IONP was compared between $4.7 \mathrm{~T}$ and $9.4 \mathrm{~T}$ (Figs. 2-3). The digitally added additional R1 was calculated from the relaxivities of $3 \mathrm{~nm}$ IONP at both field strengths equivalent to the expected R1 enhancement from 0.02, $0.04,0.06,0.08$, and $0.1 \mathrm{mM}[\mathrm{Fe}]$, resulting in $0.034,0.069,0.103,0.138$, and $0.172 \mathrm{~s}^{-1}$ at $4.7 \mathrm{~T}$ and 0.014 , 
$0.029,0.043,0.058$, and $0.072 \mathrm{~s}^{-1}$ at $9.4 \mathrm{~T}$. The hypothetical additional R1 was added to random patches in mouse cortex with small 1-8 voxel patches added to right cortex and larger 20-30 voxel patches added to left cortex, mimicking the various pathology sizes. For both low and high field, the R1 enhancements are much clearer for the larger patches (mouse brain -left side, image left side) than smaller patches (mouse brain- right side, image left side). R1 enhancements equivalent to iron concentration of 0.08 to $0.1 \mathrm{mM}$ were visible but not conspicuous in R1 maps from both $4.7 \mathrm{~T}$ and $9.4 \mathrm{~T}$ imaging (Fig. 2a-b). The same R1 enhancements were much more conspicuous in $|\Delta R 1|$ maps, with conspicuity for R1 enhancements equivalent to iron concentration of $0.06 \mathrm{mM}$ in larger patches (Fig. 2cd). The digitally added additional R1 values for $4.7 \mathrm{~T}, \mathrm{r} 1=1.87 \mathrm{mM}^{-1} \mathrm{~s}^{-1}$, are about 2.4 times of that of 9.4 $\mathrm{T}, \mathrm{r} 1=0.79 \mathrm{mM}^{-1} \mathrm{~s}^{-1}$. The intrinsic R1 of mouse brain cortex changed from $0.53 \mathrm{~s}^{-1}$ at $9.4 \mathrm{~T}$ to $0.62 \mathrm{~s}^{-1}$ at 4.7 T. Consequently, the R1 maps at 4.7 T showed better conspicuity than those of 9.4 T. However, in the I $\triangle \mathrm{R} 1$ | maps, $9.4 \mathrm{~T}$ showed similar conspicuity as $4.7 \mathrm{~T}$ to the digital simulated $\mathrm{R} 1 \mathrm{of} 3 \mathrm{~nm}$ IONP. The higher intrinsic IONP signal at $4.7 \mathrm{~T}$ and the improved reproducibility at $9.4 \mathrm{~T}$ had approximately equivalent effects, making conspicuity at $4.7 \mathrm{~T}$ and $9.4 \mathrm{~T}$ in $|\Delta \mathrm{R} 1|$ maps very similar overall. These effects were found across multiple mice at both $4.7 \mathrm{~T}$ and $9.4 \mathrm{~T}$ (Fig. 3). As expected, outlier mice having low reproducibility (mouse 5 at $4.7 \mathrm{~T}$ and mouse 10 at $9.4 \mathrm{~T}$ ) lost conspicuity to R1 enhancements equivalent to lower iron concentrations (Fig. 3.) Thus, MP2RAGE at both $4.7 \mathrm{~T}$ and $9.4 \mathrm{~T}$ were found to be equivalently appropriate approaches to assessing $3 \mathrm{~nm}$ IONP contrast agents, as long as reproducibility was good.

It is readily apparent that higher relaxivity contrast agents will be more easily detectible. In our previous report ${ }^{41}$ we predicted that the lower limit of sensitivity of MP2RAGE derived R1 maps to $3 \mathrm{~nm}$ IONP would be $0.03 \mathrm{mM}$. In the previous report, Kim et al., 2021, ${ }^{41}$, the $3 \mathrm{~nm}$ IONPs were intracranially injected into the mouse brain at 2 relatively high concentrations, $0.1 \mathrm{mM}$ and $0.25 \mathrm{mM}$ (Supplementary Fig. 3, reproduced from Fig. 8 in reference 41 ) and we extrapolated down to the $95 \%$ confidence bound for the reproducibility of the $|\Delta R 1|$ map at $9.4 \mathrm{~T}$. The $3 \mathrm{~nm}$ IONP used in our previous report had a higher relaxivity of $1.25 \mathrm{mM}^{-1} \mathrm{~s}^{-1}$ at $9.4 \mathrm{~T}$, reflecting the moderate batch-to-batch variability in producing these IONP contrast agents. We repeated the digital simulations using relaxivities equivalent to those from the $3 \mathrm{~nm}$ IONP with $\mathrm{r} 1=1.25 \mathrm{mM}^{-1} \mathrm{~s}^{-1}$. As predicted, $|\Delta \mathrm{R} 1|$ maps had good conspicuity for larger patches with enhancement equivalent to that expected from as little as $0.03 \mathrm{mM}[\mathrm{Fe}]$ of $3 \mathrm{~nm}$ IONP with $\mathrm{r} 1=1.25 \mathrm{mM}^{-}$ ${ }^{1} \mathrm{~s}^{-1}$. (Fig. 4). This result underscores the importance of optimizing the $\mathrm{r} 1$ of the contrast agents for detection of subtle pathologies or circumstances in which it is challenging to get contrast agents across the blood brain barrier.

\section{Assessment of sensitivity of R2 map on $3 \mathrm{~nm}$ IONP at 9.4 T.}

While the $3 \mathrm{~nm}$ IONPs were selected as contrast agents largely due to their R1 properties, they also may be used as R2 contrast agents. The sensitivity of $3 \mathrm{~nm}$ IONP on R2 maps was digitally simulated at $9.4 \mathrm{~T}$ (Fig. 5). Two independent in vivo mouse brain R2 maps were obtained from 3D T2 weighted images with 
repetition time $(T R)=1.0 \mathrm{~s}$. The relatively short $T R$ was chosen to obtain 3D R2 maps of whole mouse brains within a reasonable scan time (10 min total scan time for each R2 mapping); the R2 values of mouse brain were largely preserved compared to measurements made with long TR (8 s) (Supplementary Fig. 4). The transverse relaxivity (r2) of the $3 \mathrm{~nm}$ IONP at $9.4 \mathrm{~T}$ was $20.1 \mathrm{mM}^{-1} \mathrm{~s}^{-1}$. Using this $\mathrm{r} 2$, digital simulations of $|\Delta R 2|$ were performed analogously to those performed for $|\Delta R 1|$. To examine the detection limit of R2 maps for $3 \mathrm{~nm}$ IONP contrast agents, digital simulation was also performed based on hypothetical $3 \mathrm{~nm}$ IONP having low $\mathrm{r} 2,15.7 \mathrm{mM}^{-1} \mathrm{~s}^{-1}$. The digitally added additional R2 values were calculated from the r2s of $3 \mathrm{~nm}$ IONPs at 9.4 T equivalent to the expected R2 enhancement from 0.02, $0.04,0.06,0.08$, and $0.1 \mathrm{mM}$ [Fe]. Image co-registration was done prior to digital seeding of additional R2 as in the R1 digital simulations. The R2 co-registration was accurate efficient in most brain region except brain tissues around ventricle. The visibility of R2 enhancements were better from high $r 2,20.1 \mathrm{mM}^{-1} \mathrm{~s}^{-1}$, than low $r 2,15.7 \mathrm{mM}^{-1} \mathrm{~s}^{-1}$, (Fig. 5a-b). The same R2 enhancements were much more conspicuous in $|\Delta R 2|$ maps (Fig. 5c) for simulated high r2 contrast agents, whereas the R2 enhancements from $3 \mathrm{~nm}$ IONP with $\mathrm{r} 2=15.7 \mathrm{mM}^{-1} \mathrm{~s}^{-1}$ were barely visible (Fig. $5 \mathrm{~d}$ ). This showed that the intrinsically high R2 of in vivo mouse brain at $9.4 \mathrm{~T}$, which was about $25 \mathrm{~s}^{-1}$ compared to R1 of $0.53 \mathrm{~s}^{-1}$ in this study, requires contrast agents having high $\mathrm{r} 2$ to visualize R2 enhancement induced by exogenous transverse MR contrast agents. In Fig. $5 c$, the conspicuity for R2 enhancements from high transverse relaxivity, $r 2=20.1 \mathrm{mM}^{-1} \mathrm{~s}^{-1}$, were slightly less than those from R1 at 9.4 T. Thus, R2 mapping at 9.4 T provided similar conspicuity to R1 mapping if and only if the contrast agents have relatively high $r 2$. Overall, this raises the intriguing possibility that 3 nm IONPs could be used as both R1 and R2 contrast agents at high magnet field strengths.

\section{Assessment of sensitivity of quantitative human brain MRI to simulated $3 \mathrm{~nm}$ IONP at 3.0 T.}

Digital simulations were performed using data from in vivo human brain R1 maps at 3.0 T. Two independent R1 maps were obtained from a healthy adult volunteer one-week apart. Image co-registration was done as for the in vivo mouse study. The longitudinal relaxation rate constant, R1, of $3 \mathrm{~nm}$ IONP was assessed from 0.02 to $0.1 \mathrm{mM}$ [Fe] of IONP based on the longitudinal relaxivity of $3 \mathrm{~nm}$ IONP at 3.0 T, $3.94 \mathrm{mM}^{-1} \mathrm{~s}^{-1}$ (Supplementary Fig. 5) As for the in vivo mouse simulations, the hypothetical R1 enhancement from $3 \mathrm{~nm}$ IONPs was calculated using concentration $(\mathrm{mM}) \times$ relaxivity resulting in 0.0 , $0.079,0.16,0.24,0.32$, and $0.39 \mathrm{~s}^{-1}$ for $0.0,0.02,0.04,0.06,0.08$, and $0.1 \mathrm{mM}$ of [Fe] respectively. Digital simulations were performed on well-known pathologically vulnerable brain regions including the hippocampus (Fig. 6) and depths of cortical sulci (Fig. 7). The R1 enhancement was not clearly visible from the R1 map with digitally added R1 values equivalent to $0.06 \mathrm{mM}$ of [Fe] or less for hippocampus (Fig. 6d-i - g-i and Fig. 6j-i - m-i). This low conspicuity was also observed in depths of cortical sulci even with signal equivalent to $0.1 \mathrm{mM}[\mathrm{Fe}]($ Fig. 7e-i). However, the absolute R1 difference $(|\Delta \mathrm{R} 1|)$ maps had high conspicuity of R1 enhancement even with much lower simulated iron concentrations, down to 0.04 $\mathrm{mM}$ for hippocampus (Fig. 6f-ii and 6l-ii), and down to $0.06 \mathrm{mM}$ cortical sulcal depth (Fig. 7c-ii). The I $\triangle \mathrm{R} 1$ | maps following image co-registration also effectively suppressed the tissue regions having 
intrinsically high R1, providing clear conspicuity of digitally added R1 enhancement (Supplementary Fig. 6.)

As for the in vivo human brain R1 maps, digital simulations were also performed on in vivo human brain R2 maps. Two independent R2 maps were obtained within 2 hrs from the second healthy volunteer (Supplementary Fig. 7), who is different from the volunteer for the R1 map. The subject was taken out from the MR scanner after acquiring the 1st R2 map and re-positioned into MR scanner for the 2nd R2 map. The hypothetical R2 enhancement was calculated using transverse relaxivity of $3 \mathrm{~nm}$ IONP at 3.0 T, $12.9 \mathrm{mM}^{-1} \mathrm{~s}^{-1}$ (Supplementary Fig. 5), and iron concentration resulting in $0.0,0.26,0.52,0.77,1.03,1.29$, 2.59 , and $3.89 \mathrm{~s}^{-1}$ for $0.0,0.02,0.04,0.06,0.08,0.1,0.2$, and $0.3 \mathrm{mM}$ of [Fe] respectively. The digital simulations were performed on both hippocampus and depths of cortical sulci (Fig. 8). Unlike the digital simulation results from R1 map, the R2 enhancement was not apparent for the absolute R2 difference (I $\triangle \mathrm{R} 2$ |) maps for the iron concentration below $0.1 \mathrm{mM}$. Higher simulated iron concentrations, between 0.1 to $0.3 \mathrm{mM}$ [Fe], were needed to visualize the R2 enhancement. Overall, this shows that $3 \mathrm{~nm}$ IONPs would provide more sensitive detection of pathology when used as R1 contrast agents at $3.0 \mathrm{~T}$.

\section{Discussion}

In this study, we described an approach to assessing the enhancing effect of proposed longitudinal (R1) and transverse (R2) MR contrast agents in both human and mouse brain. We used digital simulation in this study, which avoids physical damage and can be done even without the availability of effective BBB crossing methods. To simulate the hypothetical in vivo condition, the proposed digital simulation method involved two independent R1 or R2 maps from the same subject within a short time interval, one week or less in this study, to minimize physiology-induced changes. After co-registration of the two independent R1 or R2 maps and digital seeding of simulated changes on the second map, the absolute difference map between them clearly showed the hypothetical enhancement that would be expected from the $3 \mathrm{~nm}$ IONP contrast agent at various concentrations. Overall, the digital simulations provided quantitative analyses for prediction of the sensitivity of candidate MRI contrast agents in brain tissues.

Importantly, the use of difference maps substantially improved the conspicuity of the simulated effects of contrast agent for both in vivo mouse and human brain. It is also likely that routine T1 or T2 weighted imaging, for which baseline subtraction is not routinely employed, will be less sensitive than R1 or R2 mapping-based approaches where the baseline subtraction can be readily performed. Thus, R1 or R2 mapping at pre- and post-injection combined with image co-registration could be a preferred approach to quantitatively detect MR contrast agents like $3 \mathrm{~nm}$ IONPs. Interestingly, $3 \mathrm{~nm}$ IONPs showed both R1 and R2 enhancing effects, indicating its potential role as a dual contrast agent. This suggests that acquiring both R1 and R2 maps would provide improved reliability in assessing tissue localization of the enhancement by $3 \mathrm{~nm}$ IONP MR contrast agents.

Besides factors relating to the MR contrast agent itself, the proposed digital simulation methodology heavily depends on the reproducibility of the R1 and R2 maps. In this study, the reproducibility was 
quantitatively presented using absolute difference maps at a voxel-by-voxel level. Alternatively, the testretest signal to noise ratio (TrTSNR) is also applicable to provide quantitative reproducibility of these imaging methodologies as in our previous report ${ }^{41}$. Thus, reporting the voxel-by-voxel reproducibility of the MR methodology for all MR based biomarker studies is recommended. Another critical imaging related factor is the image co-registration. The quality of co-registration will determine the overall sensitivity of the employed MR contrast agents, especially when the pathology is patchy or sparse. Importantly, the co-registration quality depends on both raw data reproducibility and spatial resolution. There have been extensive efforts towards the development of improved MR hardware including multichannel RF coil, high power imaging gradients, and high magnet field for human scanner. The improvements of MR imaging hardware will continue to improve the spatial resolution and ultimately image co-registration quality. One advantage of the R1 mapping over the R2 mapping in this study was the improved co-registration accuracy for the R1 maps; R2 map co-registration suffered from artifacts in the peri-ventricular region, which was not the case for R1 maps.

Our current approach has certain limitations regarding measuring the true R1 or R2 enhancement of $3 \mathrm{~nm}$ IONPs in brain tissue. First, our approach is based on assumption that all brain tissues have the same amount of R1 or R2 enhancement from the $3 \mathrm{~nm}$ IONPs, which might not be true in vivo. Importantly, the delivery of the contrast agent to the target in vivo has not been addressed. The amount of $3 \mathrm{~nm}$ IONPs delivered to specific brain regions will vary depending on the approaches used to confer BBB crossing and molecular specificity. Furthermore, the half-life of $3 \mathrm{~nm}$ IONPs in brain may also affect the R1 or R2 enhancement if its chemical properties change over time. Additionally, it is unknown how long IONPS would remain in the brain. Future studies are planned to address these questions.

In conclusion, the proposed digital simulation provides a useful approach to quantitatively assess the MR signal conspicuity for candidate MRI relaxation contrast agents in humans and mice. The same approach should be applicable to any quantitative MRI like T2 star or T1 rho. In addition, the proposed approach can provide the reliability of proposed alternative MRI acquisition methodologies. Thus, the digital simulation approach will be beneficial for the development of both MRI contrast agents and MRI methodologies.

\section{Methods}

\section{Relaxivity of $3 \mathrm{~nm}$ IONPs at 3.0 T, 4.7 T, and 9.4 T}

$3 \mathrm{~nm}$ IONPs were synthesized following the methods of Kim et al. and validated using dynamic light scattering and electron microscopy ${ }^{42}$. Synthesis and ligand exchange were performed at the Chemistry and Synthesis Center (CSC) in NIH. Iron chloride hexahydrate (FeCl3.6H2O), 80\% oleic acid, Polyethylene glycol methyl ether (PEG750), tetrahydrofuran (THF) and dichloromethane were purchased from Sigma Aldrich. Sodium oleate was purchased from Tokyo Chemical Industry (Portland, Oregon, USA). HydroxylPEG-Azide 2k (OH-PEG-N3) was obtained from Biochempeg. Iron concentrations were measured by inductively coupled plasma optical emission spectroscopy (ICP-OES) method using Agilent 5900 SVDV 
ICP-OES (Santa Clara, California, USA). A series of phantoms were made from $3 \mathrm{~nm}$ IONPs suspended in $1 \mathrm{x}$ PBS and diluted to $0.02,0.04,0.06,0.08$, and $0.1 \mathrm{mM}[\mathrm{Fe}]$. 1xPBS served as a control. Both longitudinal and transverse relaxation rates of $3 \mathrm{~nm}$ IONP were assessed at 3.0 T, 4.7 T, and 9.4 T magnetic field.

The longitudinal relaxation rate constant $\left(R 1,1 / T 1, s^{-1}\right)$ of $3 \mathrm{~nm}$ IONP was assessed using modified fast inversion recovery (MFIR) ${ }^{43}$. The inversion MR data was collected after passing the null point to suppress macromolecule effects on R1 estimation ${ }^{43,44}$. The repetition time (TR) was $5.0 \mathrm{~s}$ for $3.0 \mathrm{~T}$ and $8.0 \mathrm{~s}$ for both $4.7 \mathrm{~T}$ and $9.4 \mathrm{~T}$ where the echo time (TE) was $3.0 \mathrm{~ms}$ for all magnetic field strength. The inversion delay time points were optimized for each magnetic field as follows; 1) $3.0 \mathrm{~T}-13$ inversion delay time points at $500,750,1000,1250,1500,1750,2000,2250,2500,3000,3500,4000$, and $4500 \mathrm{~ms}$. 2) $4.7 \mathrm{~T}-$ 17 inversion delay time points at $800,900,1000,1100,1200,1300,1600,1900,2300,2600,2900,3300$, $3600,3900,5500,6500,7900 \mathrm{~ms}$. 3) $9.4 \mathrm{~T}-15$ inversion delay time points at, $1100,1200,1300,1600$, $1900,2300,2600,2900,3300,3600,3900,5000,6000,7000,7900$ ms. The R1 was estimated using Bayesian analysis toolbox (http://bayesiananalysis.wustl.edu/). The longitudinal relaxivity, $\mathrm{r} 1\left(\mathrm{mM}^{-1} \mathrm{~s}^{-1}\right)$, of $3 \mathrm{~nm}$ IONP was estimated from the R1 of $3 \mathrm{~nm}$ IONP at five different iron concentration, 0.02 to 0.1 $\mathrm{mM}[\mathrm{Fe}]$. The obtained $\mathrm{r} 1\left(\mathrm{mM}^{-1} \mathrm{~s}^{-1}\right)$ was used to calculate the theoretical R1 enhancement by $\mathrm{r} 1\left(\mathrm{mM}^{-}\right.$ $\left.{ }^{1} \mathrm{~s}^{-1}\right) \in[\mathrm{Fe}](\mathrm{mM})$.

The transverse relaxation rate constant ( $\left.R 2, \mathrm{~s}^{-1}, 1 / \mathrm{T} 2\right)$ of $3 \mathrm{~nm}$ IONPs was assessed using multi echo train MR sequence, 24 ms echo spacing and 20 echo train at both $3.0 \mathrm{~T}$ and $9.4 \mathrm{~T}$. The TR was $5.0 \mathrm{~s}$ for 3.0 $\mathrm{T}$ and $8.0 \mathrm{~s}$ for $9.4 \mathrm{~T}$. The R2 was estimated using Bayesian analysis toolbox (http://bayesiananalysis.wustl.edu/). Similar to $\mathrm{r} 1$, the transverse relaxivity, $r 2\left(\mathrm{mM}^{-1} \mathrm{~s}^{-1}\right)$, of $3 \mathrm{~nm}$ IONP was estimated from the R2 of IONP at five different iron concentration, 0.02 to $0.1 \mathrm{mM}[\mathrm{Fe}]$.

\section{In vivo subjects}

Human brain MRI images were performed under the National Institutes of Health Institutional Review Board (NIH IRB) approved protocol (NCT00001711). Informed consent was obtained prior to a health subject R1 mapping MR scans, which were conducted in the Clinical Center at the National Institutes of Health $(\mathrm{NIH})$. Human brain MRI image sets for R2 mapping were acquired from a health subject following informed consent at the National Intrepid Center of Excellence. All human related procedures and methods were performed in accordance with the relevant guidelines and regulations.

All animal experiments were conducted under protocols approved by the National Institute of Neurological Disorders and Stroke (NINDS)/ National Institute on Deafness and Other Communication Disorders (NIDCD) Animal Care and Use Committee in the NIH Clinical Center. All in vivo animal related procedures and methods were performed in accordance with the protocols approved by the Institutional Animal Care and Use Committee (IACUC) at the $\mathrm{NIH}$ and were in accordance with ARRIVE guidelines (https://arriveguidelines.org). C57BL6 female mice at 10 weeks of age were purchased from Jackson 
labs and used at 12-weeks of age. A total 11 mice were used in this study, five mice at $4.7 \mathrm{~T}$ and six mice at 9.4 T. Among the 6 mice scanned at 9.4 T, five mice, (mouse ID 06-10), were from our previous report ${ }^{41}$.

\section{In vivo MRI of mouse brain at 4.7 T and 9.4 T}

In vivo mouse brain MR scans were performed on Bruker 4.7 T and 9.4 T scanner (Bruker, Ettlingen, Germany) with imaging gradient $180 \mathrm{mT} / \mathrm{m}$ for $4.7 \mathrm{~T}$ and $260 \mathrm{mT} / \mathrm{m}$ for $9.4 \mathrm{~T}$ on Paravision 6.01 platform. All employed naïve mice underwent two independent MR scans, MP2RAGE or multi echo T2 scan, within one-week.

At 4.7 T, a single channel RF coil was used to acquire MP2RAGE data at $160 \mu \mathrm{m} \times 160 \mu \mathrm{m} \times 480 \mu \mathrm{m}$ voxel size, zero-filled to $80 \mu \mathrm{m} \times 80 \mu \mathrm{m} \times 80 \mu \mathrm{m}$, covering the entire brain with 8 averages and 60 minutes total scan time. The thick image slice was selected to increase signal to noise ratio at magnetic field, $4.7 \mathrm{~T}$. The MP2RAGE imaging parameters were TR/TE/TI1/TI2 (ms) = 8000/3/1100/2600, 9-degree flip angle, and 640 ms segment duration with 2 inversion for a k-space plane. In vivo mouse brain R2 map at 4.7 T was not pursued due to the limitation of gradient maximum rise time (or slew rate).

At 9.4 T, 4-channel RF coil used to acquire MP2RAGE data at $160 \mu \mathrm{m} \times 160 \mu \mathrm{m} \times 160 \mu \mathrm{m}$ voxel size, zerofilled to $80 \mu \mathrm{m} \times 80 \mu \mathrm{m} \times 80 \mu \mathrm{m}$, covering the entire brain with 6 averages and 95 minutes total scan time. The MP2RAGE imaging parameters were TR/TE/TI1/TI2 (ms) = 8000/3/1300/3600, 9-degree flip angle, and 640 ms segment duration with 2 inversion for a k-space plane. The MR protocol of MP2RAGE at 9.4 $\mathrm{T}$ is the same as our previous report ${ }^{41}$. The $\mathrm{T} 1$ maps of in vivo mouse brain was estimated from raw MP2RAGE and converted into R1 maps. In addition, multi echo T2 weighted images were acquired at 160 $\mu \mathrm{m} \times 160 \mu \mathrm{m} \times 240 \mu \mathrm{m}$ voxel size, zero-filled to $80 \mu \mathrm{m} \times 80 \mu \mathrm{m} \times 80 \mu \mathrm{m}$, covering the entire brain with TR/ echo spacing/ echo train number/ average/ total scan time $=8.0 \mathrm{~s} / 8.0 \mathrm{~ms} / 20 / 1 / 10$ minutes. The R2 map was estimated using the Bayesian analysis toolbox (http://bayesiananalysis.wustl.edu/). The mouse brain T1 (1/R1) was $1.88 \mathrm{~s}$ in the current study. Thus, the short TR might cause T1 contamination on mouse brain R2 map. The R2 value of in vivo mouse brain at 9.4 T dependence on TR was examined by comparing 2D MR sequence having long TR (8.0 s) with 3D having short TR (1.0 s), see

\section{Supplementary Fig. 4.}

\section{In vivo MRI of human brain at $3.0 \mathrm{~T}$}

Two different 3.0 T human scanners were used for in vivo human brain MR scan with two normal adult subjects. In vivo human brain MP2RAGE MR scans were performed on a Siemens Prisma $3.0 \mathrm{~T}$ magnet (Erlangen, Germany) with $80 \mathrm{mT} / \mathrm{m}$ imaging gradient and 32 channel radio frequency (RF) coil. The first normal adult subject underwent two independent 3-dimensional MP2RAGE scans within one-week, at the same time of the day on each session. The MP2RAGE images were acquired with the following parameters: echo time $(\mathrm{TE})=2.88 \mathrm{~ms}$, repetition time $(\mathrm{TR})=5000 \mathrm{~ms}$, inversion delay times $\mathrm{TI} / \mathrm{T} / 2=$ 
$700 / 2500 \mathrm{~ms}$, field of view $($ FOV $)=256 \times 256 \times 192 \mathrm{~mm}$, matrix $=256 \times 256 \times 192$, flip angles $=4$-degree for TI1 and 5-degree for TI2, band width $=240 \mathrm{hz} /$ pixel, GRAPPA acceleration factor $=3$, total scan time $=8$ minutes 56 second. The obtained MP2RAGE data had $1.0 \times 1.0 \times 1.0 \mathrm{~mm}$ isotropic voxel size. The T1 maps of in vivo human brain were estimated from raw MP2RAGE data using the vendor providing imaging handling software, Syngo MR D13D. The obtained T1 maps were converted into R1 maps (= $1 / \mathrm{T} 1)$.

In vivo human brain $\mathrm{T} 2$ mapping scan using $\mathrm{T} 2$ weighted imaging (T2 $\mathrm{WI}$ ) sequence were performed on a GE SIGNA 3.0 T magnet (Waukesah, Milwaukee, USA) with $80 \mathrm{mT} / \mathrm{m}$ imaging gradient and 32 channel radio frequency $(\mathrm{RF})$ coil. The second normal adult subject underwent two independent T2 mapping MR scans within 2 hours. The second subject took a short break between the 1 st and 2nd MR scan. 2dimensional T2WI were acquired with the following parameters: TR $=4200 \mathrm{~ms}, \mathrm{FOV}=240 \mathrm{~mm} \times 240 \mathrm{~mm}$, matrix $=256 \times 256$, slice thickness $=1.2 \mathrm{~mm}$, total number of slices $=142, \mathrm{TE}=30 \mathrm{~ms}$ and $110 \mathrm{~ms}$, total scan time $=12$ minutes 32 seconds. T2 map was calculated using linear T2 MR signal decay approach with two echo time (TE) MR signals following the methods from a previous report ${ }^{45}$. The $30 \mathrm{~ms}$ echo time was selected as short echo time T2WI to avoid myelin water effect in T2 map calculation ${ }^{46}$ and resulted in improved reproducibility (Supplementary Fig. 7).. The $110 \mathrm{~ms}$ echo time was selected as long echo time T2WI ensuring about $50 \%$ signal to noise ratio of $30 \mathrm{~ms}$ echo time T2WI. The resulting T2 map was converted into R2 maps (=1/T2).

\section{Data and image processing}

For both 4.7 T and 9.4 T, two MP2RAGE data were acquired from the same mouse within one-week time frame producing two independent R1 maps. Using the advanced normalization tools (ANTs, http://stnava.github.io/ANTs/ ${ }^{47}$, the 2nd R1 map was co-registered to the 1st R1 map. After coregistration the absolute $\Delta \mathrm{R} 1(|\Delta \mathrm{R} 1|)$ map between 1st $\mathrm{R} 1$ map and the $2 \mathrm{nd} \mathrm{R} 1$ map co-registered to $1 \mathrm{st}$ R1 map was calculated, producing a baseline. The hypothesized R1 enhancing effects on in vivo mouse brain induced by $3 \mathrm{~nm}$ IONPs were simulated by digitally adding R1 values to the 2nd R1 map coregistered to 1st R1 map. This approach simulates the result that could hypothetically be obtained if a subject were scanned at baseline, administered an IONP contrast agent, and then scanned again at an appropriate time. The hypothetical R1 enhancement of the $3 \mathrm{~nm}$ IONPs in mouse brain was calculated from equation [1].

\section{$\Delta \mathrm{R} 1=\mathrm{r} 1\left(\mathrm{mM}^{-1} \mathrm{~s}^{-1}\right) \times[\mathrm{Fe}](\mathrm{mM})[1]$}

The digital simulated additional R1 values were randomly placed in both small (from 1 to 8 voxels) and larger (from 20 to 30 voxels) zones in the cortex of the 2nd R1 map co-registered to 1st R1 map. It was assumed that all brain tissues have the same degree of R1 enhancing effect from the same concentrations of $3 \mathrm{~nm}$ IONP. This procedure was performed for five hypothetical IONP iron concentrations: $0.02,0.04,0.06,0.08$, and $0.1 \mathrm{mM}$. Similar to the baseline $|\Delta R 1|$ map, the $|\Delta R 1|$ maps between the 1st R1 map and the co-registered 2nd R1 map having the hypothesized effects of $3 \mathrm{~nm}$ IONP 
were calculated to assess the sensitivity of MP2RAGE derived R1 maps to $3 \mathrm{~nm}$ IONP contrast agents. In vivo mouse brain R2 maps underwent the same procedures where the hypothetical R2 enhancement of the $3 \mathrm{~nm}$ IONPs in mouse brain was calculated from equation [2].

\section{$\Delta \mathrm{R} 2=\mathrm{r} 2\left(\mathrm{mM}^{-1} \mathrm{~s}^{-1}\right) \times[\mathrm{Fe}](\mathrm{mM})[2]$}

The same procedures were performed on in vivo human brain R1 maps obtained at 3.0 T. Following image co-registration, the baseline $|\Delta R 1|$ was obtained. The digital simulated additional $R 1$ values, which were calculated from the 1 of the $3 \mathrm{~nm}$ IONPs at $3.0 \mathrm{~T}$, were randomly placed in both small (from 1 to 2 voxels) and larger (from 3 to 9 voxels) zones in representative human brain regions. The $|\Delta R 1|$ map between the 1st R1 map and co-registered 2nd R1 map after digitally added R1 equivalent to several concentrations of $3 \mathrm{~nm}$ IONP were calculated. Analogous procedures were conducted using R2 maps.

\section{Declarations}

\section{Acknowledgements}

This research was supported by the Intramural Research Program of the NIH, NINDS. Additional support was provided by the Uniformed Services University of the Health Sciences. We thank Drs. Alan Koretsky, Duong Nguyen, Simone Mastrogiacomo, Mr. Thomas J. Esparza, Ms. Shiran Su, and the Laboratory of Functional and Molecular Imaging for stimulating discussions. In addition, we thank Drs. Grant Bonavia, Wei Liu, and Mr. Adam Clifton for the human brain R2 map protocol discussion. We thank Dr. Mark Whiting for editorial assistance.

\section{Disclaimer}

The views expressed here are those of the authors, and do not represent those of the $\mathrm{NIH}$, Uniformed Services University of Health Sciences, Department of Defense, or other government agency.

\section{Author contributions}

Joong Kim conducted in vivo mouse brain MR experiments, performed data analyses, and wrote the first draft of the manuscript. Wen-Tung Wang conducted in vivo human brain MR experiments and in vitro phantom MR experiments. Andrew Knutsen built the pipeline for image co-registration and optimized the image co-registration parameters. David Brody conceived and designed the study, obtained funding, and contributed to manuscript preparation. All authors reviewed manuscript.

\section{Disclosures:}


DLB has performed paid consulting for Pfizer, Health Advances, Signum Nutralogix, Kypha, iPerian, Sage Therapeutics, St Louis Public Defenders Office, Avid Radiopharmaceuticals (Eli Lilly), Intellectual Ventures. DLB holds Equity: Inner Cosmos LLC. DLB received royalties for sales of Concussion Care Manual (Oxford University Press). DLB receives honoraria from Mary Ann Liebert, Inc. Publisher of Journal of Neurotrauma for services as Editor-in-Chief. DLB is a member of the editorial board of Acta Neuropathologica. None of these constitute a conflict of interest.

\section{References}

1. Zhang, H. et al. Age-specific optimization of T1-weighted brain MRI throughout infancy. Neurolmage, 199, 387-395 https://doi.org/10.1016/j.neuroimage.2019.05.075 (2019).

2. Seiger, R. et al. Voxel-based morphometry at ultra-high fields. a comparison of 7T and 3T MRI data. Neurolmage, 113, 207-216 https://doi.org/10.1016/j.neuroimage.2015.03.019 (2015).

3. Jovicich, J. et al. Brain morphometry reproducibility in multi-center 3T MRI studies: a comparison of cross-sectional and longitudinal segmentations. Neurolmage, 83, 472-484 https://doi.org/10.1016/j.neuroimage.2013.05.007 (2013).

4. Falkovskiy, P. et al. Comparison of accelerated T1-weighted whole-brain structural-imaging protocols. Neurolmage, 124, 157-167 https://doi.org/10.1016/j.neuroimage.2015.08.026 (2016).

5. Haacke, E. M., Xu, Y., Cheng, Y. C. \& Reichenbach, J. R. Susceptibility weighted imaging (SWI). Magnetic resonance in medicine, 52, 612-618 https://doi.org/10.1002/mrm.20198 (2004).

6. Labadie, C. et al. Myelin water mapping by spatially regularized longitudinal relaxographic imaging at high magnetic fields. Magnetic resonance in medicine, 71, 375-387 https://doi.org/10.1002/mrm.24670 (2014).

7. Kolind, S. H., Madler, B., Fischer, S., Li, D. K. \& MacKay, A. L. Myelin water imaging: Implementation and development at 3.0T and comparison to 1.5T measurements. Magnetic resonance in medicine, 62, 106-115 https://doi.org/10.1002/mrm.21966 (2009).

8. Misaki, M. et al. Contrast enhancement by combining T1- and T2-weighted structural brain MR Images. Magnetic resonance in medicine, 74, 1609-1620 https://doi.org/10.1002/mrm.25560 (2015).

9. Pagnozzi, A. M., Fripp, J. \& Rose, S. E. Quantifying deep grey matter atrophy using automated segmentation approaches: A systematic review of structural MRI studies. Neurolmage, 201, 116018 https://doi.org/10.1016/j.neuroimage.2019.116018 (2019).

10. Wen, J., Cross, A. H. \& Yablonskiy, D. A. On the role of physiological fluctuations in quantitative gradient echo MRI: implications for GEPCI, QSM, and SWI. Magnetic resonance in medicine, 73, 195203 https://doi.org/10.1002/mrm.25114 (2015).

11. Gabr, R. E. et al. Interleaved susceptibility-weighted and FLAIR MRI for imaging lesion-penetrating veins in multiple sclerosis. Magnetic resonance in medicine, 80, 1132-1137 https://doi.org/10.1002/mrm.27091 (2018). 
12. Zivadinov, R. et al. Abnormal subcortical deep-gray matter susceptibility-weighted imaging filtered phase measurements in patients with multiple sclerosis: a case-control study. Neurolmage, 59, 331339 https://doi.org/10.1016/j.neuroimage.2011.07.045 (2012).

13. Maranzano, J. et al. Automated separation of diffusely abnormal white matter from focal white matter lesions on MRI in multiple sclerosis. Neurolmage, 213, 116690 https://doi.org/10.1016/j.neuroimage.2020.116690 (2020).

14. de Sitter, A. et al. Performance of five research-domain automated WM lesion segmentation methods in a multi-center MS study. Neurolmage, $163,106-114$ https://doi.org/10.1016/j.neuroimage.2017.09.011 (2017).

15. Eibofner, F., Steidle, G., Kehlbach, R., Bantleon, R. \& Schick, F. Positive contrast imaging of iron oxide nanoparticles with susceptibility-weighted imaging. Magnetic resonance in medicine, 64, 1027-1038 https://doi.org/10.1002/mrm.22498 (2010).

16. Stroh, A. et al. In vivo detection limits of magnetically labeled embryonic stem cells in the rat brain using high-field (17.6 T) magnetic resonance imaging. Neurolmage, 24, 635-645 https://doi.org/10.1016/j.neuroimage.2004.09.014 (2005).

17. Vreys, R. et al. MRI visualization of endogenous neural progenitor cell migration along the RMS in the adult mouse brain: validation of various MPIO labeling strategies. Neurolmage, 49, 2094-2103 https://doi.org/10.1016/j.neuroimage.2009.10.034 (2010).

18. Peng, Y. K., Tsang, S. C. E. \& Chou, P. T. Chemical design of nanoprobes for T1-weighted magnetic resonance imaging. Materials today, 19, 336-348 (2016).

19. Gupta, A. K. \& Gupta, M. Synthesis and surface engineering of iron oxide nanoparticles for biomedical applications., 26, 3995-4021 https://doi.org/10.1016/j.biomaterials.2004.10.012 (2005).

20. Xie, W. et al. Shape-, size- and structure-controlled synthesis and biocompatibility of iron oxide nanoparticles for magnetic theranostics. Theranostics, 8, 3284-3307 https://doi.org/10.7150/thno.25220 (2018).

21. Marques, J. P. et al. MP2RAGE, a self bias-field corrected sequence for improved segmentation and T1-mapping at high field. Neurolmage, 49, 1271-1281 https://doi.org/10.1016/j.neuroimage.2009.10.002 (2010).

22. Manuel, A., Li, W., Jellus, V., Hughes, T. \& Prasad, P. V. Variable flip angle-based fast three-dimensional T1 mapping for delayed gadolinium-enhanced MRI of cartilage of the knee: need for B1 correction. Magnetic resonance in medicine, 65, 1377-1383 https://doi.org/10.1002/mrm.22720 (2011).

23. Zhang, J. et al. Quantifying iron-oxide nanoparticles at high concentration based on longitudinal relaxation using a three-dimensional SWIFT Look-Locker sequence. Magnetic resonance in medicine, 71, 1982-1988 https://doi.org/10.1002/mrm.25181 (2014).

24. Ma, D. et al. Magnetic resonance fingerprinting. Nature, 495, 187-192 https://doi.org/10.1038/nature11971 (2013).

25. Dong, X. Current Strategies for Brain Drug Delivery. Theranostics, 8, 1481-1493 https://doi.org/10.7150/thno.21254 (2018). 
26. Zhou, Y., Peng, Z., Seven, E. S. \& Leblanc, R. M. Crossing the blood-brain barrier with nanoparticles. J Control Release, 270, 290-303 https://doi.org/10.1016/j.jconrel.2017.12.015 (2018).

27. Xie, J., Shen, Z., Anraku, Y., Kataoka, K. \& Chen, X. Nanomaterial-based blood-brain-barrier (BBB) crossing strategies., 224, 119491 https://doi.org/10.1016/j.biomaterials.2019.119491 (2019).

28. Kariolis, M. S. et al. Brain delivery oftherapeutic proteins using an Fc fragment blood-brain barrier transport vehicle in mice and monkeys. SCIENCE TRANSLATIONAL MEDICINE, 12, 1-13 https://doi.org/10.1126/scitranslmed.aay1359 (2020).

29. Kovacs, Z. I. et al. Disrupting the blood-brain barrier by focused ultrasound induces sterile inflammation. Proc Natl Acad Sci U S A, 114, E75-E84 https://doi.org/10.1073/pnas.1614777114 (2017).

30. Savas, J. N. et al. Amyloid Accumulation Drives Proteome-wide Alterations in Mouse Models of Alzheimer's Disease-like Pathology. Cell Rep, 21, 2614-2627 https://doi.org/10.1016/j.celrep.2017.11.009 (2017).

31. Wang, A., Das, P., Switzer, R. C. 3, Golde, T. E., Jankowsky, J. L. \& rd, \& Robust amyloid clearance in a mouse model of Alzheimer's disease provides novel insights into the mechanism of amyloid-beta immunotherapy. J Neurosci, 31, 4124-4136 https://doi.org/10.1523/JNEUROSCI.5077-10.2011 (2011).

32. Mouzon, B. et al. Repetitive mild traumatic brain injury in a mouse model produces learning and memory deficits accompanied by histological changes. J Neurotrauma, 29, 2761-2773 https://doi.org/10.1089/neu.2012.2498 (2012).

33. Guo, L. et al. Dynamic rewiring of neural circuits in the motor cortex in mouse models of Parkinson's disease. Nat Neurosci, 18, 1299-1309 https://doi.org/10.1038/nn.4082 (2015).

34. Geula, C. Abnormalities of neural circuitry in Alzheimer's disease: hippocampus and cortical cholinergic innervation. Neurology, 51, S18-29 discussion S65-

17https://doi.org/10.1212/wnl.51.1_suppl_1.s18 (1998).

35. Cendes, F., Sakamoto, A. C., Spreafico, R., Bingaman, W. \& Becker, A. J. Epilepsies associated with hippocampal sclerosis. Acta Neuropathol, 128, 21-37 https://doi.org/10.1007/s00401-014-1292-0 (2014).

36. Berg, A. T., Pardoe, H. R., Fulbright, R. K., Schuele, S. U. \& Jackson, G. D. Hippocampal size anomalies in a community-based cohort with childhood-onset epilepsy. Neurology, 76, 1415-1421 https://doi.org/10.1212/WNL.0b013e318216712b (2011).

37. Wohlsein, P. et al. Spontaneous human herpes virus type 1 infection in a chinchilla (Chinchilla lanigera f. dom.). Acta Neuropathol, 104, 674-678 https://doi.org/10.1007/s00401-002-0597-6 (2002).

38. McKee, A. C. et al. Chronic traumatic encephalopathy in athletes: progressive tauopathy after repetitive head injury. J Neuropathol Exp Neurol, 68, 709-735 https://doi.org/10.1097/NEN.0b013e3181a9d503 (2009). 
39. Ghajari, M., Hellyer, P. J. \& Sharp, D. J. Computational modelling of traumatic brain injury predicts the location of chronic traumatic encephalopathy pathology., 140, 333-343

https://doi.org/10.1093/brain/aww317 (2017).

40. Holleran, L. et al. Axonal disruption in white matter underlying cortical sulcus tau pathology in chronic traumatic encephalopathy. Acta Neuropathol, 133, 367-380 https://doi.org/10.1007/s00401-017-1686-x (2017).

41. Kim, J. H. et al. Sensitive detection of extremely small iron oxide nanoparticles in living mice using MP2RAGE with advanced image co-registration. Sci Rep, 11, 106 https://doi.org/10.1038/s41598020-80181-9 (2021).

42. Kim, B. H. et al. Large-scale synthesis of uniform and extremely small-sized iron oxide nanoparticles for high-resolution T1 magnetic resonance imaging contrast agents. Journal of the American Chemical Society, 133, 12624-12631 https://doi.org/10.1021/ja203340u (2011).

43. Gupta, R. K., Ferretti, J. A., Becker, E. D. \& Weiss, G. H. A modified fast inversion-recovery technique for spin-lattice relaxation measurements. Journal of Magnetic Resonance, 38, 447-452 (1980).

44. Meinerz, K. et al. Bayesian Modeling of NMR Data: Quantifying Longitudinal Relaxation in Vivo, and in Vitro with a Tissue-Water-Relaxation Mimic (Crosslinked Bovine Serum Albumin). Appl. Magn. Reson, 49, 3-24 (2018).

45. Hasan, K. M., Walimuni, I. S., Kramer, L. A. \& Narayana, P. A. Human brain iron mapping using atlasbased T2 relaxometry. Magnetic resonance in medicine, 67, 731-739 https://doi.org/10.1002/mrm.23054 (2012).

46. MacKay, A. L. \& Laule, C. Magnetic Resonance of Myelin Water: An in vivo Marker for Myelin. Brain Plast, 2, 71-91 https://doi.org/10.3233/BPL-160033 (2016).

47. Avants, B. B., Epstein, C. L., Grossman, M. \& Gee, J. C. Symmetric diffeomorphic image registration with cross-correlation: evaluating automated labeling of elderly and neurodegenerative brain. Med Image Anal, 12, 26-41 https://doi.org/10.1016/j.media.2007.06.004 (2008).

\section{Figures}



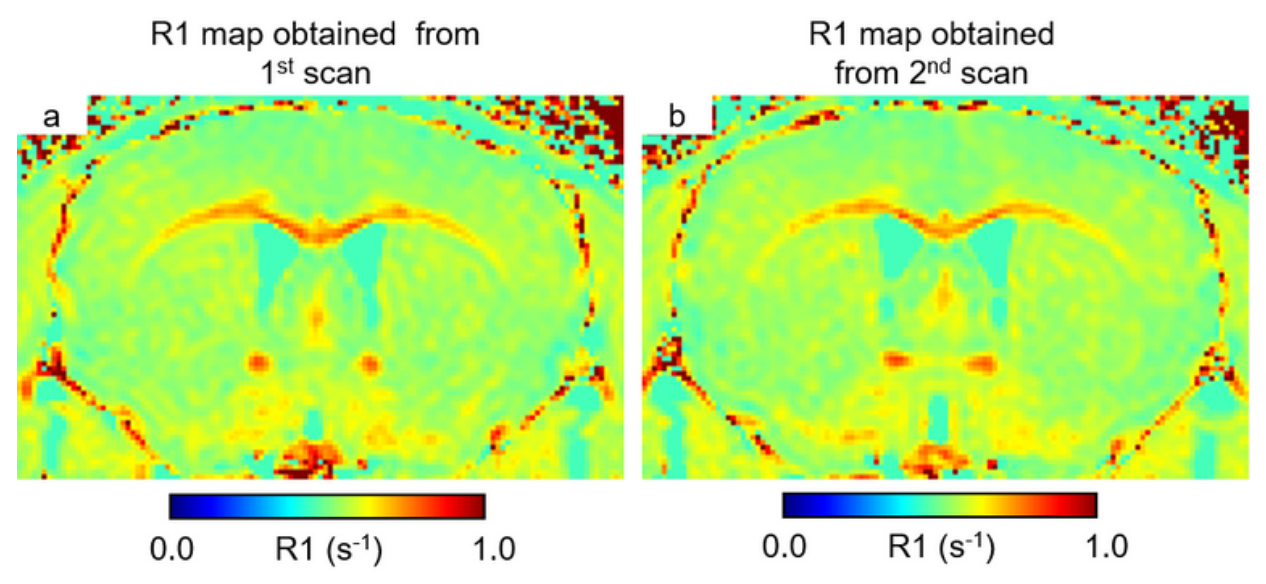

$|\Delta R 1|$

Difference between a and $c$

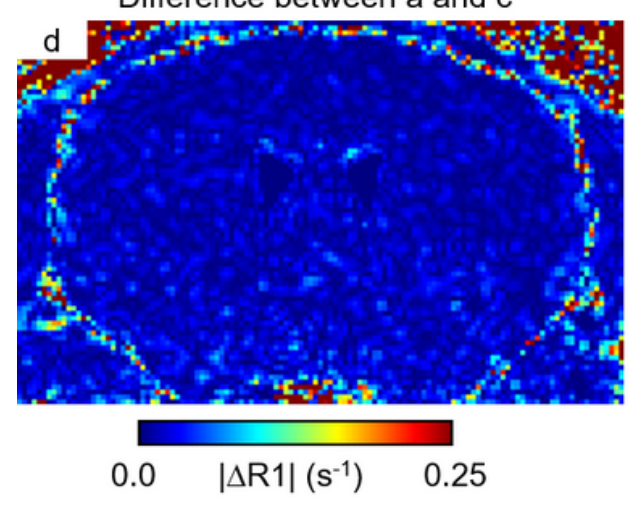

Digital seeding of IONP R1 on $2^{\text {nd }} \mathrm{R} 1$ map co-registered to the $1^{\text {st }} \mathrm{R} 1$ map
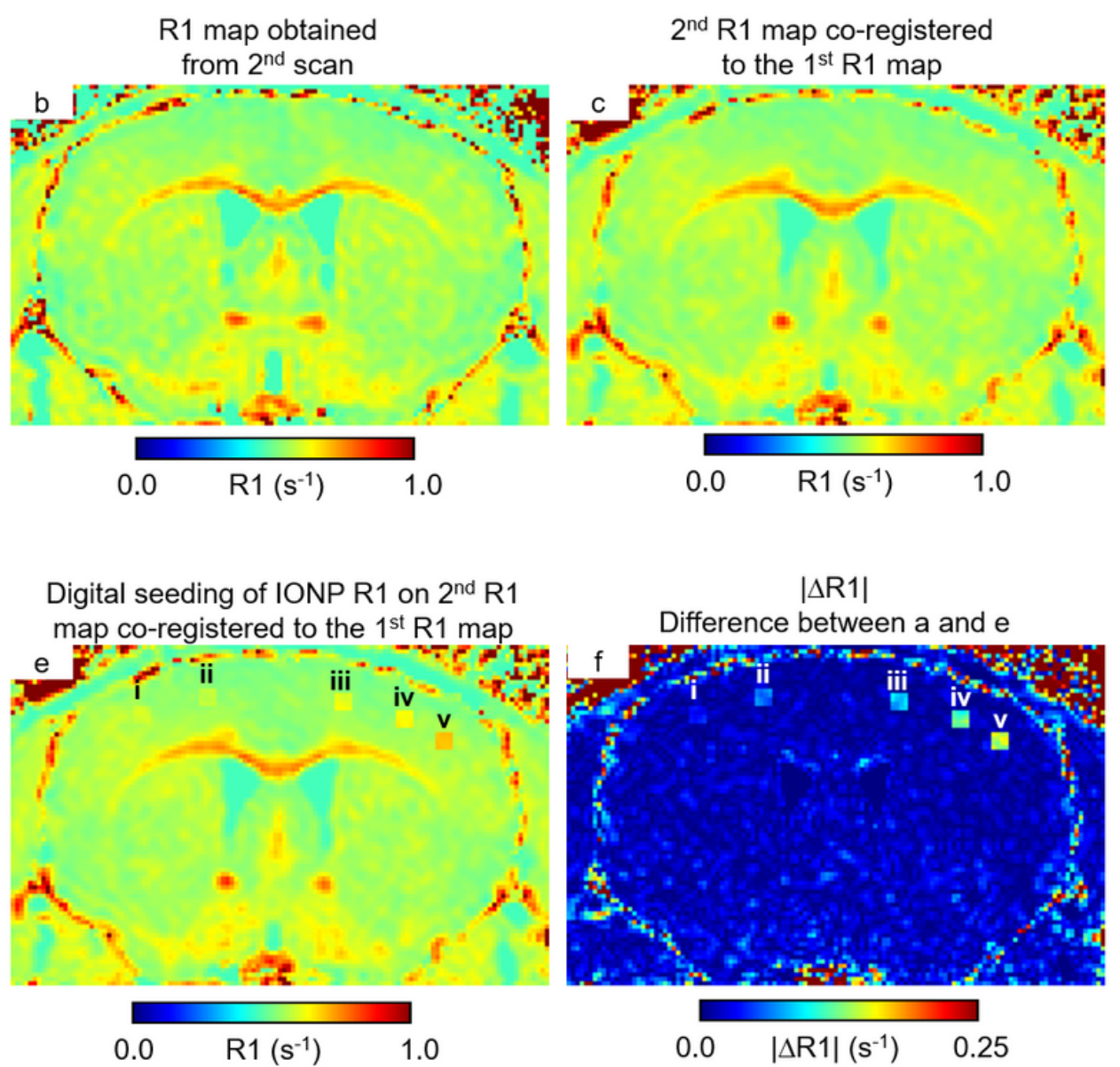

$|\Delta \mathrm{R} 1|$

Difference between a and $\mathrm{e}$

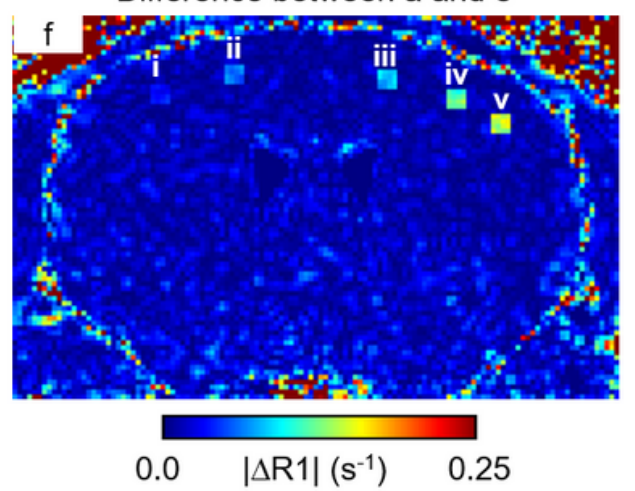

\section{Figure 1}

The digital simulation workflow. a, b. two independent R1 maps (1st and 2nd R1 map) from the same mouse, obtained one week apart. c. 2nd R1 map co-registered to 1st R1 map using ANTs. $d$. the absolute $\Delta \mathrm{R} 1(|\Delta \mathrm{R} 1|)$ map between 1st (panel a) and co-registered 2nd (panel c) R1 map. e. The simulated effects on R1 of IONP contrast agent were digitally added to panel $\mathrm{c}$ in 5 regions (i-v): the digitally added R1 values were 0.0265 (i), 0.053 (ii), 0.0795 (iii), 0.106 (iv), and 0.1325 (v) s-1. f. the $|\Delta R 1|$ map between 1st (panel a) and co-registered 2nd R1 map with digitally simulated R1 effects of IONPs (panel e). The locations with digitally simulated additional $R 1$ are clearly visible in $|\Delta R 1|$ without interference from intrinsic R1 value differences. 

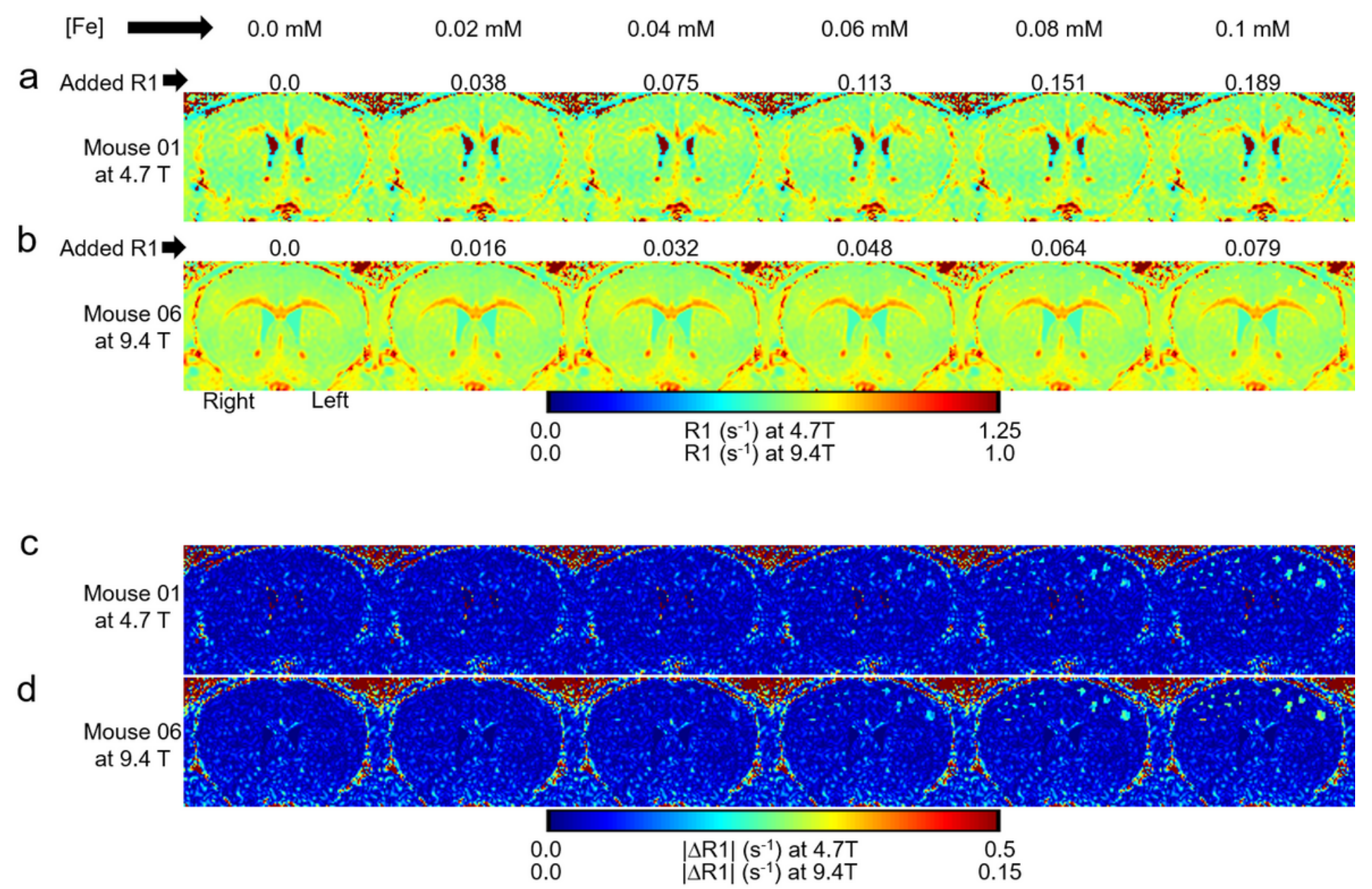

Figure 2

In vivo mouse brain $\mathrm{R} 1$ maps after digital simulation of additional $\mathrm{R} 1$ and the absolute $\Delta \mathrm{R} 1(|\Delta \mathrm{R} 1|)$ maps for low (4.7 T) and high (9.4 T) magnetic field. a. Coronal R1 maps with digitally added additional R1 values equivalent to 0.02 to $0.1 \mathrm{mM}$ [Fe] IONPs at $4.7 \mathrm{~T}$. b. Coronal R1 maps with digitally added additional R1 values equivalent to 0.02 to $0.1 \mathrm{mM}[\mathrm{Fe}]$ IONPs at $9.4 \mathrm{~T}$. c. $|\Delta \mathrm{R} 1|$ maps at $4.7 \mathrm{~T}$. d. $|\Delta \mathrm{R} 1|$ maps at $9.4 \mathrm{~T}$. Note that the intensity scales of $9.4 \mathrm{~T}$ are different from those of $4.7 \mathrm{~T}$. 
a

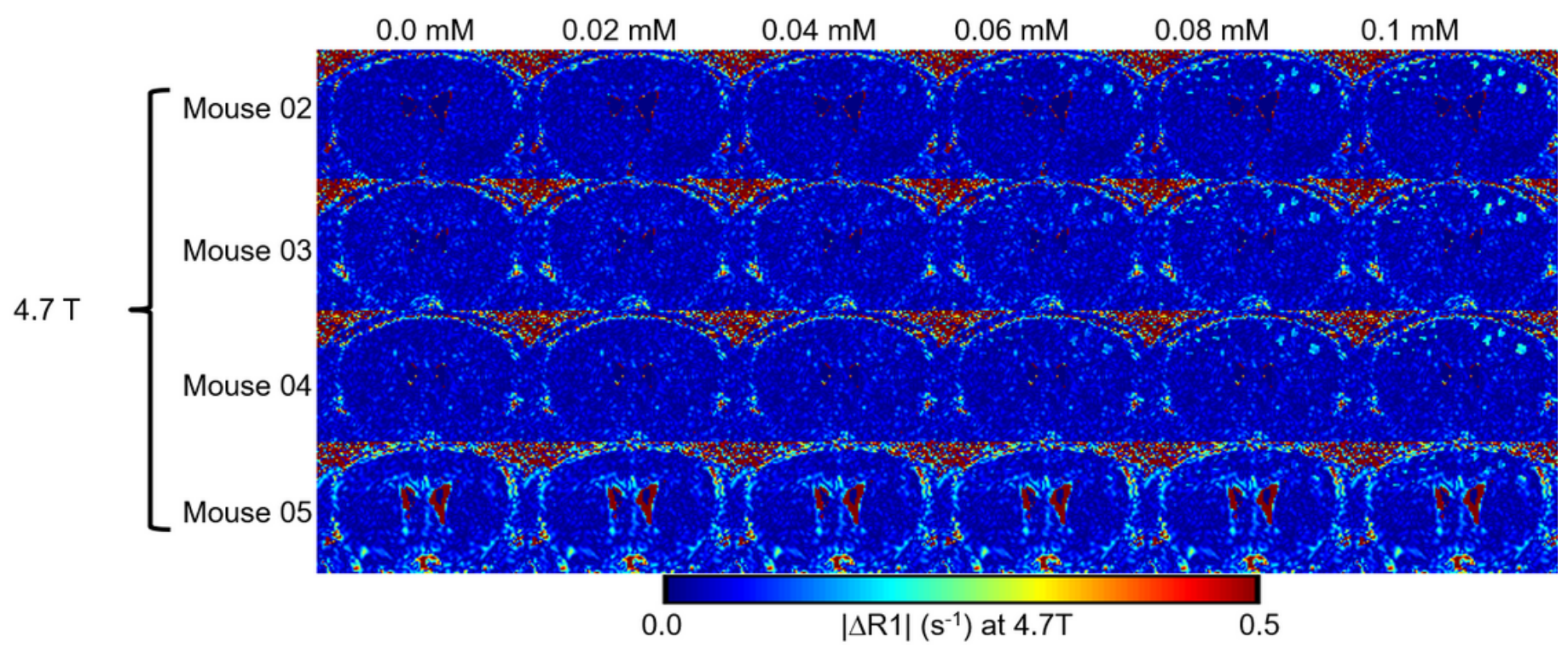

b

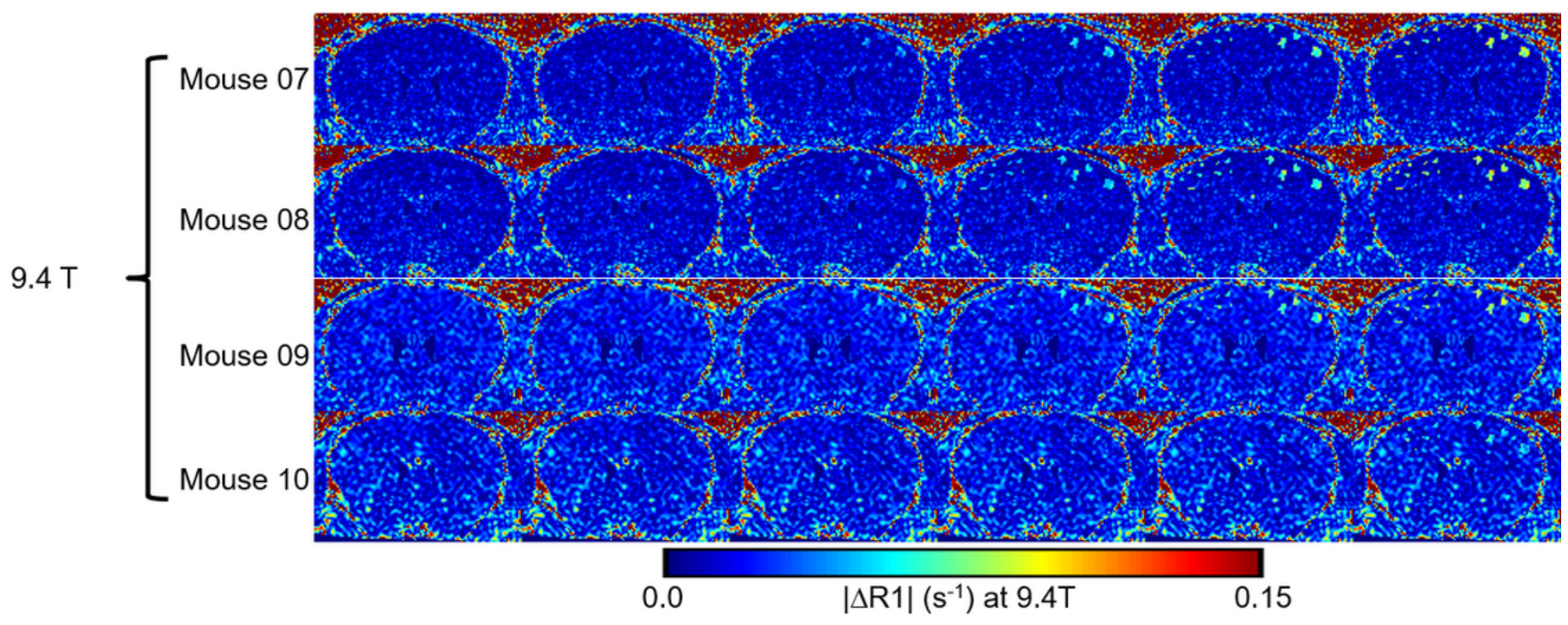

\section{Figure 3}

Absolute $\triangle \mathrm{R} 1(|\Delta \mathrm{R} 1|)$ maps showing sensitivity to simulated $3 \mathrm{~nm}$ IONP at low $(4.7 \mathrm{~T})$ and high $(9.4 \mathrm{~T})$ magnetic field. a. $|\Delta R 1|$ maps at $4.7 \mathrm{~T}$ for four mice, mouse $02-05$. b. $|\Delta R 1|$ maps at $9.4 T$ for four mice, mouse $07-10$. Note that the intensity scale of $9.4 \mathrm{~T}$ is different from those of $4.7 \mathrm{~T}$ due to the higher reproducibility of R1 mapping at $9.4 \mathrm{~T}$. Individual mice with lower intrinsic reproducibility, mouse 05 and mouse 10 , lost sensitivity to simulated $3 \mathrm{~nm}$ IONPs at both low and high magnet field. 


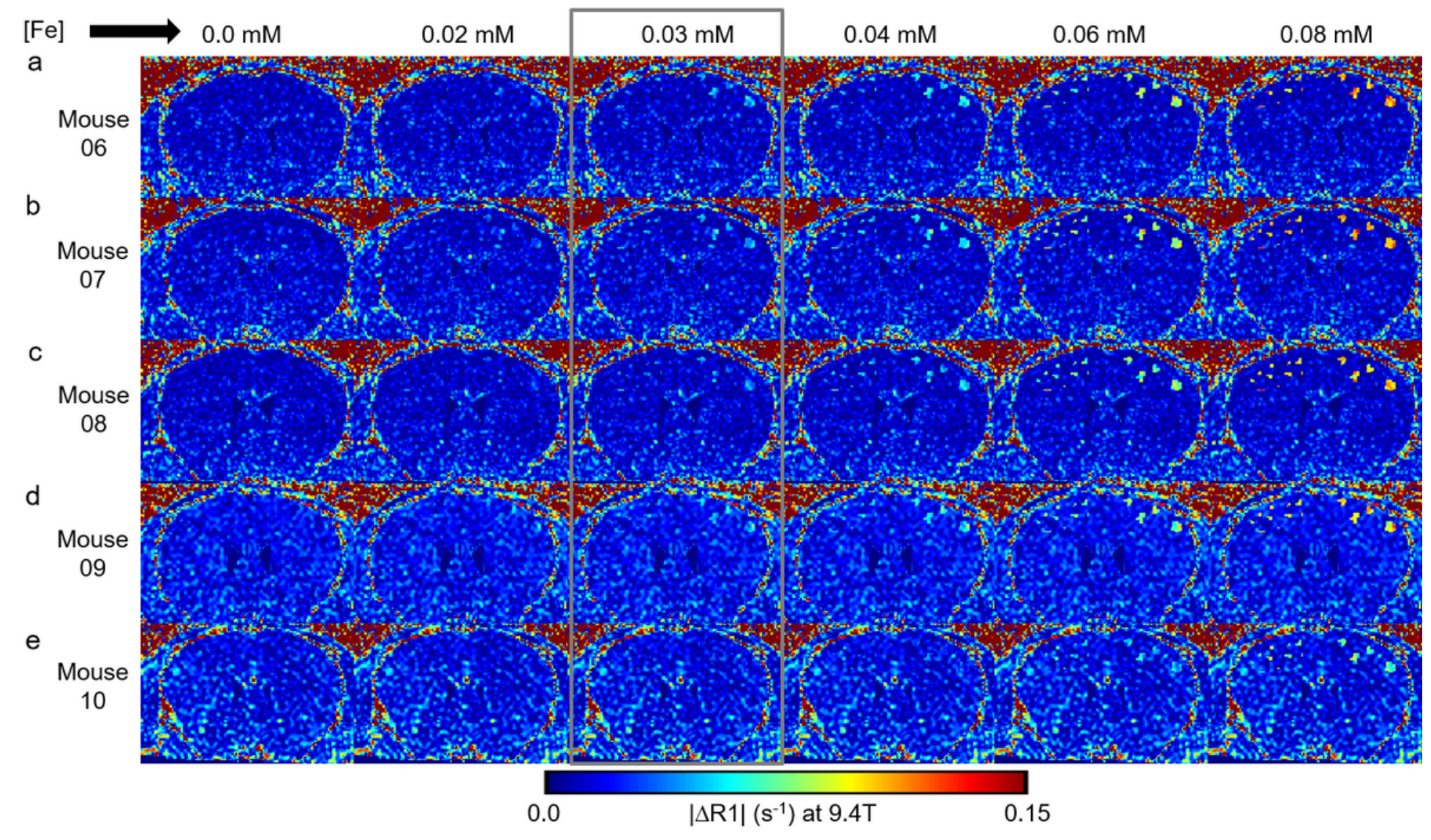

\section{Figure 4}

Absolute $\triangle \mathrm{R} 1(|\Delta \mathrm{R} 1|)$ maps from digital simulation of $3 \mathrm{~nm}$ IONP with improved $\mathrm{r} 1=1.25 \mathrm{mM}-1 \mathrm{~s}-1 \mathrm{on}$ in vivo mouse brain at $9.4 \mathrm{~T}$. a - e. $|\Delta \mathrm{R} 1|$ maps at $9.4 \mathrm{~T}$ for five mice, mouse 06 - 10. Digital simulation was done on mouse 06 - 10 using r1 = 1.25 mM-1s-1 to simulate the effect of IONPs with improved r1. Note that the highest iron concentration is $0.08 \mathrm{mM}$. The digital simulation of $3 \mathrm{~nm}$ IONP using high relaxivity, $\mathrm{r} 1=1.25 \mathrm{mM}-1 \mathrm{~s}-1$, showed better conspicuity than those of low relaxivity, $0.72 \mathrm{mM}-1 \mathrm{~s}-1$, See panel b of Figure 3. The digitally simulated R1 enhancement is visible to $0.03 \mathrm{mM}$ iron concentration for mouse 06 - 09, as predicted from our previous report, see Supplementary Figure 3 or Figure 8 of reference 41. 
a

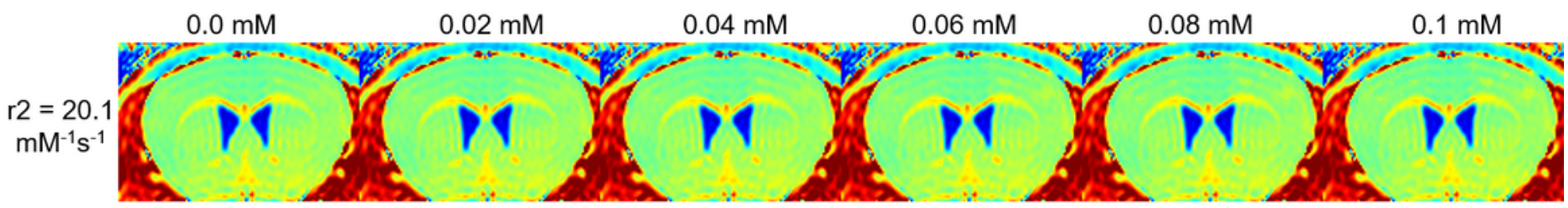

b
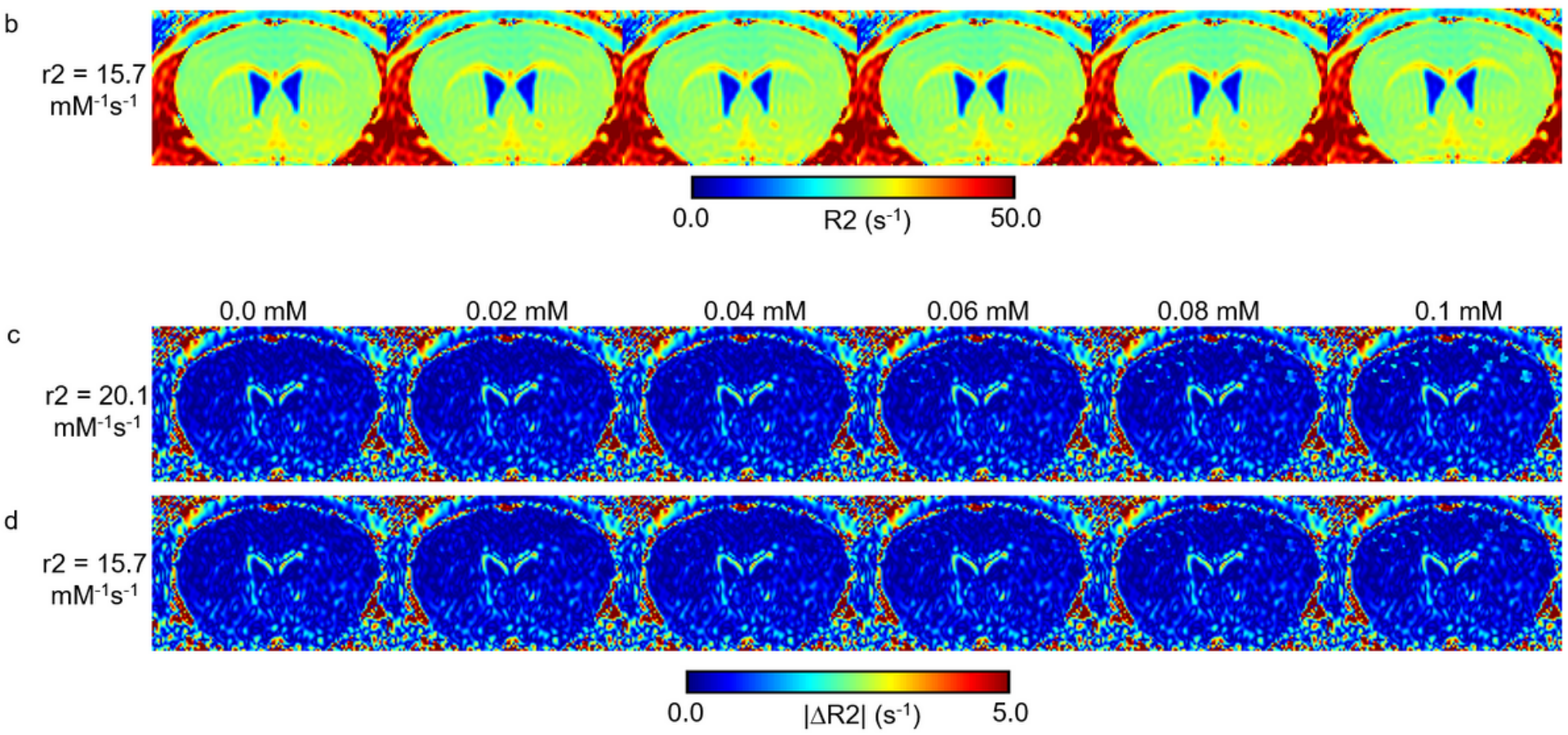

Figure 5

In vivo mouse brain $\mathrm{R} 2$ maps with digital simulated $\mathrm{R} 2$ effects of IONPs and absolute $\Delta \mathrm{R} 2(|\Delta \mathrm{R} 2|)$ maps at 9.4 T. a. Coronal R2 maps with digitally added additional R2 values equivalent to 0.02 to $0.01 \mathrm{mM}$ [Fe] $3 \mathrm{~nm}$ IONPs with $\mathrm{r} 2=20.1 \mathrm{mM}-1 \mathrm{~s}-1$. b. Coronal R2 map having digitally added additional R2 value, $\mathrm{r} 2=$ $15.7 \mathrm{mM}-1 \mathrm{~s}-1$. The digitally added $\mathrm{R} 2$ values were displayed as iron concentration of $3 \mathrm{~nm}$ IONP for each column. c. $|\Delta \mathrm{R} 2|$ maps for $\mathrm{r} 2=20.1 \mathrm{mM}-1 \mathrm{~s}-1$. d. $|\Delta \mathrm{R} 2|$ maps for $\mathrm{r} 2=15.7 \mathrm{mM}-1 \mathrm{~s}-1$. In vivo MR scans were performed with $160 \mu \mathrm{m} \times 160 \mu \mathrm{m} \times 240 \mu \mathrm{m}$ voxel size, zero-filled to $80 \mu \mathrm{m} \times 80 \mu \mathrm{m} \times 80 \mu \mathrm{m}$ for R2 map. The digitally added R2 enhancements are more conspicuous in $|\triangle R 2|$ map than $R 2$ map and large voxels mimicking large, clustered pathology have better conspicuity than small voxels. The digital simulation of $3 \mathrm{~nm}$ IONP using high relaxivity, r2 $=20.1 \mathrm{mM}-1 \mathrm{~s}-1$, showed better conspicuity than those of low relaxivity, $15.7 \mathrm{mM}-1 \mathrm{~s}-1$. 


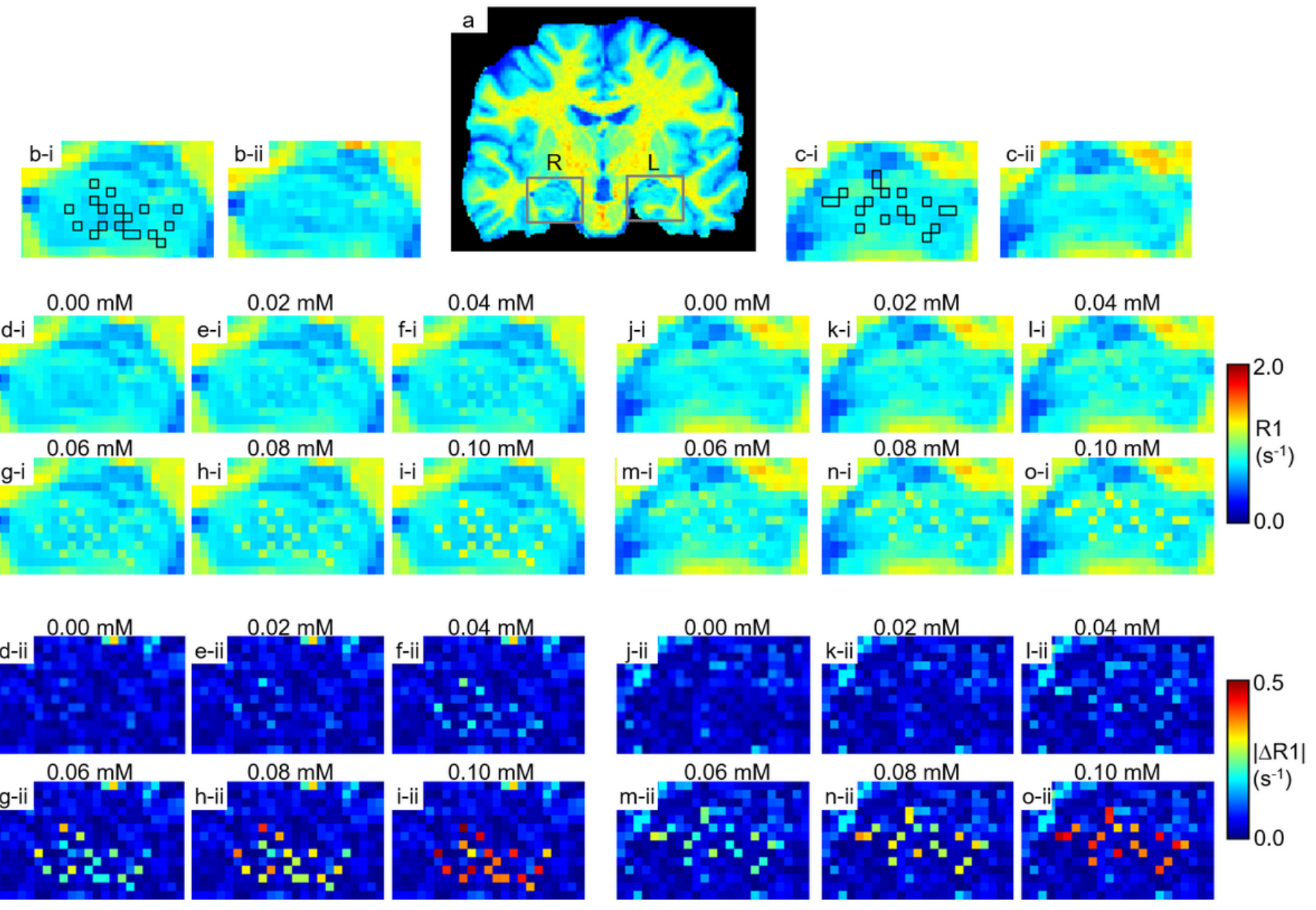

Figure 6

Digital simulation of scattered IONP R1 contrast in human hippocampus. a. Coronal R1 map from a normal human volunteer showing the location of the hippocampi. b-i. right, and c-i. left hippocampi showing the random locations for one or two voxel digital simulation additions of R1 (small boxes). b-ii, c-ii. showing the baseline scans (1st R1 map) of right (panel b-ii) and left hippocampi (panel c-ii). d-i - o-i. 2nd R1 maps in the hippocampi with digital simulated additional R1 equivalent to 0 through $0.10 \mathrm{mM}[\mathrm{Fe}]$ IONPs. The 2nd R1 map was co-registered to the 1st R1 map prior to digital simulation. d-ii - o-ii. The absolute $\Delta R 1(|\Delta R 1|)$ maps. Single voxels in hippocampus are conspicuous at $R 1$ equivalent to $0.04 \mathrm{mM}$ [Fe] or higher in the subtraction maps, but much less prominent without baseline scan subtraction. 

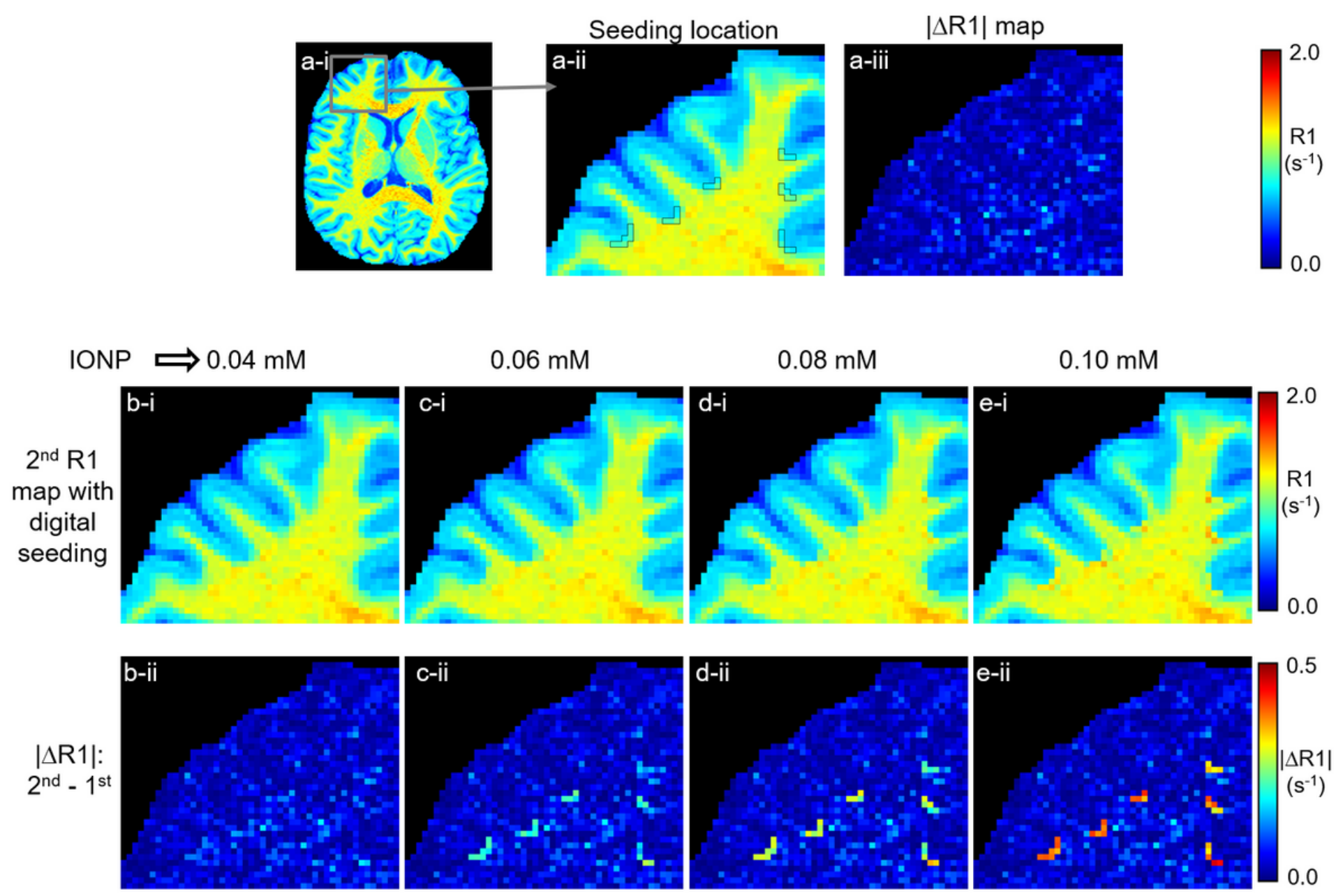

\section{Figure 7}

Digital simulation of clustered IONP R1 contrast at the depths of cortical sulci. a-i. Axial R1 map from a normal human volunteer showing the location of the frontal cortex. a-ii. Close up view of 2nd R1 map showing the random locations for 4-7 voxel digital simulation additions of R1 (boxes) at the depths of cortical sulci. a-iii. Close up view of the absolute $|\Delta R 1|$ map without addition of R1. The 2nd R1 map was co-registered to the 1st R1 map prior to digital simulation. b-i - e-i. R1 maps in the cortex with digital simulated additional R1 equivalent to 0.04 through $0.10 \mathrm{mM}$ [Fe] IONPs. b-ii - e-ii. The absolute $|\Delta R 1|$ maps. Clustered voxels in cortical sulci are prominently conspicuous at R1 equivalent to $0.06 \mathrm{mM}$ [Fe] or higher in the $|\Delta R 1|$ maps. The additions of $\mathrm{R} 1$ are essentially indistinct without subtracting co-registered baseline scans due to the higher R1 in white matter compared with gray matter. 

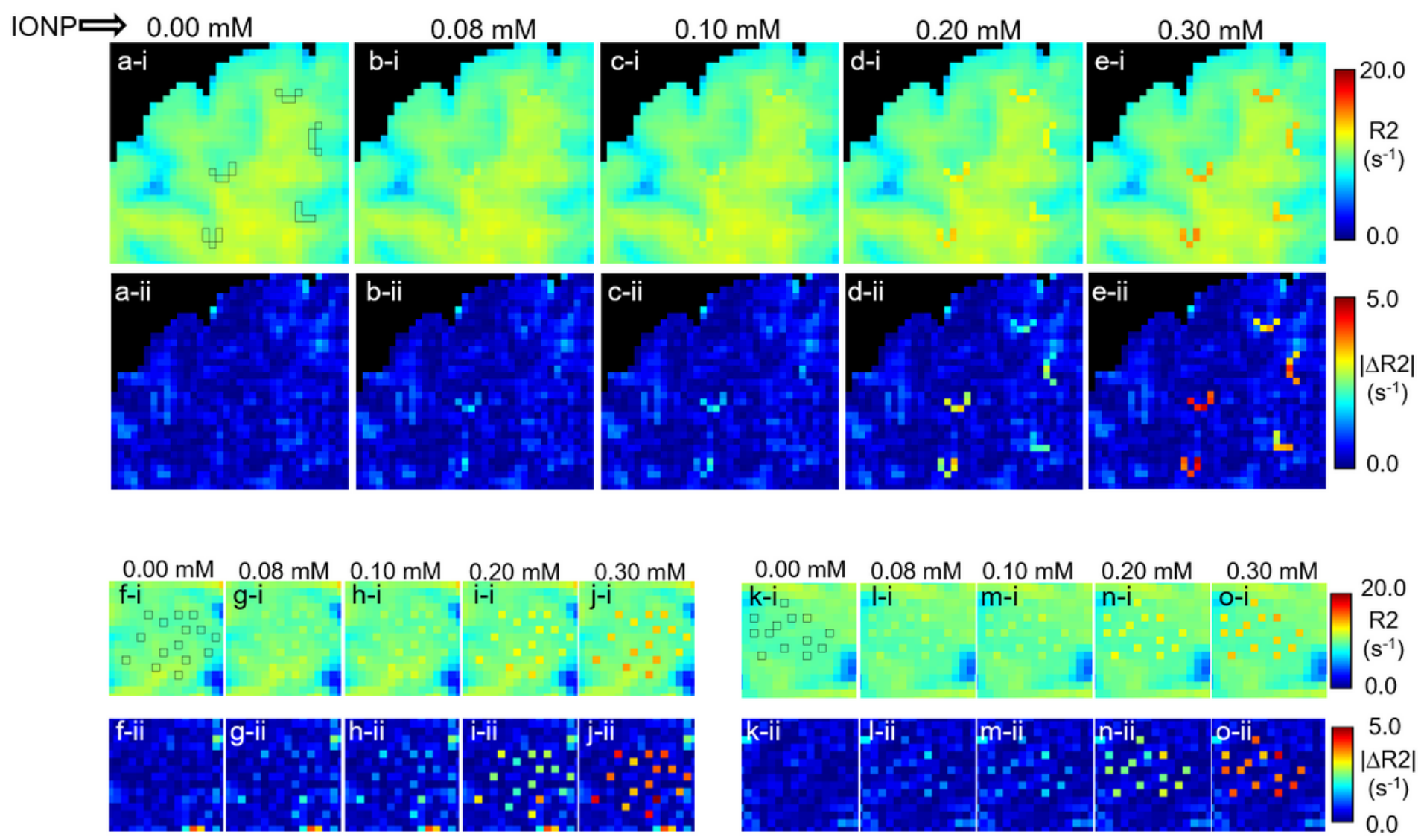

\section{Figure 8}

Digital simulation of IONP R2 contrast in human brain. The cortical sulci (a-i), left (f-i) and right (k-i) hippocampi showing location of digital simulation seeding sites. b-i - e-i. Axial R2 maps with digitally added additional R2 values equivalent to $0.08-0.3 \mathrm{mM}$ [Fe] $3 \mathrm{~nm}$ IONPs with $\mathrm{r} 2=12.9 \mathrm{mM}-1 \mathrm{~s}-1$. a-ii $-\mathrm{e}-$ ii. the absolute $\triangle \mathrm{R} 2(|\Delta \mathrm{R} 2|)$ maps of cortical sulci with additional $\mathrm{R} 2$ equivalent to $0.08-0.3 \mathrm{mM}[\mathrm{Fe}]$ IONP. g-i - j-i and l-i - o-i. coronal R2 maps with digitally added additional R2 values equivalent to 0.08 $0.3 \mathrm{mM}[\mathrm{Fe}] 3 \mathrm{~nm}$ IONPs with $\mathrm{r} 2=12.9 \mathrm{mM}-1 \mathrm{~s}-1$. $\mathrm{f}-\mathrm{ii}-\mathrm{j}-\mathrm{ii}$ and $\mathrm{k}-\mathrm{ii}-\mathrm{o}-\mathrm{ii}$. the absolute $\Delta \mathrm{R} 2(|\Delta \mathrm{R} 2|)$ maps of hippocampi with additional R2 equivalent to $0.08-0.3 \mathrm{mM}$ [Fe] IONP. Panels a-ii, $\mathrm{f}-\mathrm{ii}$, and $\mathrm{k}-\mathrm{ii}$ are the baseline absolute $\Delta \mathrm{R} 2(|\Delta \mathrm{R} 2|)$ maps without additional digital simulation. The simulated additions of R2 are barely visible at $0.1 \mathrm{mM}$ [Fe] IONP for both hippocampus and cortical sulci where the conspicuity is better at higher [Fe] IONP.

\section{Supplementary Files}

This is a list of supplementary files associated with this preprint. Click to download.

- DigitalSimulationSupplement20211stSubmission.docx 